\title{
Nodular tertiary syphilis in an immunocompetent patient ${ }^{*}$
}

\author{
Maraya de Jesus Semblano Bittencourt ${ }^{1}$ \\ Bianca Angelina Macêdodo Nascimento ${ }^{1}$ \\ Marion Guimarães Drago ${ }^{1}$
}

\author{
Arival Cardoso de Brito ${ }^{1}$ \\ Alessandra Haber Carvalho ${ }^{1}$
}

DOI: http://dx.doi.org/10.1590/abd1806-4841.20163837

\begin{abstract}
Acquired syphilis can be divided into primary, secondary, latent, and tertiary stages. About $25 \%$ of patients with untreated primary syphilis will develop late signs that generally occur after three to five years, with involvement of several organs. The authors present an immunocompetent female who developed a tertiary stage syphilis presenting with long-standing nodular plaques.
\end{abstract}

Keywords: Sexually transmitted diseases; Syphilis, cutaneous; Treponemal infections

\section{INTRODUCTION}

Syphilis is an infectious disease, sexually transmitted, caused by Treponema pallidum. ${ }^{1}$ Its incidence has been reduced with the introduction of penicillin for its treatment, however it has been sharply increasing with the advent of human immunodeficiency virus (HIV) infection. ${ }^{1,2}$ Approximately $25 \%$ of the patients with untreated primary syphilis develop symptoms of tertiary syphilis. ${ }^{1-4}$ They usually occur three to five years after primary infection and involve several organs, mainly skin, heart and central nervous system. ${ }^{3}$ In the antibiotic era, it is extremely rare to find cases of tertiary syphilis. This fact, added to the scarcity of reports in the literature in recent years, has caused lack of familiarity with clinical and histopathological aspects of tertiarism.

\section{CASE REPORT}

A 40-year-old woman had been presenting, in the last two years and six months, nodular lesions of progressive growth in the upper limbs, without associated symptoms or previous treatments. She denied any compatible symptoms or signs of primary and secondary phases or recurrence of syphilis. There was no history of injectable drug use, blood transfusion or convulsions. The dermatological examination showed extensive centrifugal-growth erythemato-violaceous nodular plaques in the upper limbs (Figure 1).

Anatomopathological examination of left arm biopsy showed atrophic epidermis and vacuolar interface dermatitis. The dermis and the subcutaneous were filled with a mixed, dense and diffuse inflammatory infiltrate, composed of lymphocytes, histio-

Received on 14.07.2014.

Approved by the Advisory Board and accepted for publication on 09.01.2015.

* Work performed at Dermatologic clinic of Universidade Federal do Pará (UFPA) - Belém (PA), Brazil.

Financial Support: None.

Conflict of Interest: None.

1 Universidade Federal do Pará (UFPA) - Belém (PA), Brazil.

@2016 by Anais Brasileiros de Dermatologia 
cytes and abundant plasmocytes. The vascular alterations included vascular proliferation and clogged vessels, with tumescent endothelium and walls infiltrated by inflammatory cells, besides erythrocyte extravasation. The infiltrate was separated from the epidermis by a grenz band (Figure 2). Caseous necrosis was not noticed. Fite-Faraco and Grocott stainings were negative for BAAR and fungi, respectively.

Due to histological suspicion of syphilis, FTA-ABS and VDRL were requested, which resulted as reagent (VDRL in a titer of 1:32). Anti-HIV 1 and 2 were negative. Patient was submitted to treatment with benzathine penicillin $(2,400,000$ IU per week, for
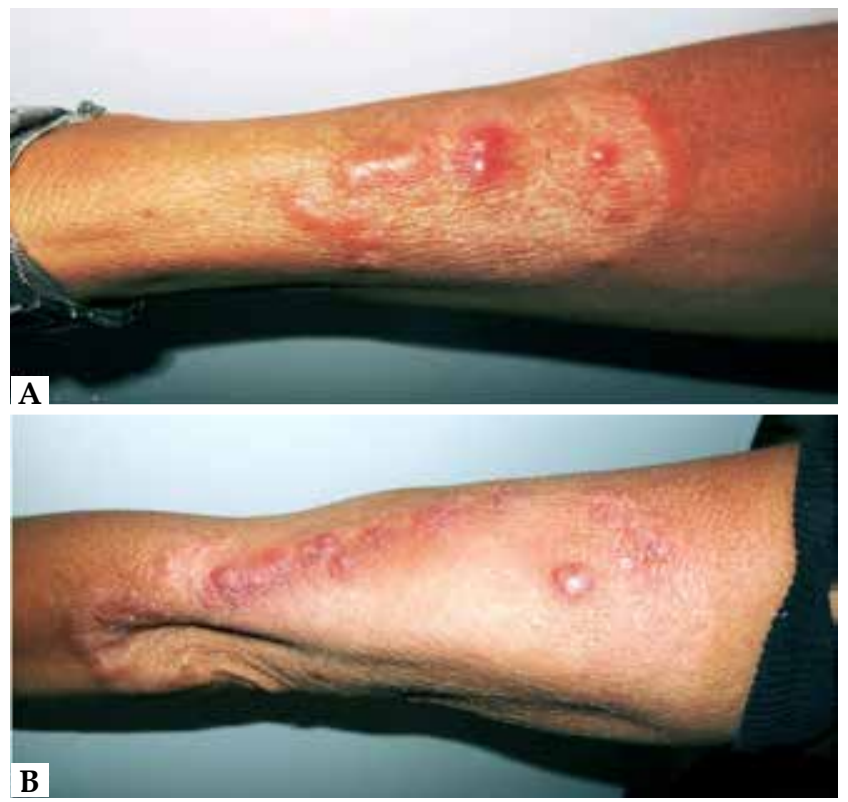

FiguRE 1: Arcuate papulonodular lesions in the right forearm (A) and left arm (B) three weeks), with complete remission of lesions (Figure 3). Electrocardiogram, echocardiogram, X-ray of long bones, eye examination, ultrasound of the abdomen, blood count and tests of hepatic and renal function showed no alterations.

\section{DISCUSSION}

Tertiary or late syphilis is a rare systemic disease which may present mucocutaneous, cardiac, ophthalmic, neurological or osseous involvement. ${ }^{1,3}$ Skin is the most affected organ, and its clinical presentation varies according to the level of involvement, which may be hypodermic (syphilitic gumma) or dermoepidermal (pso-
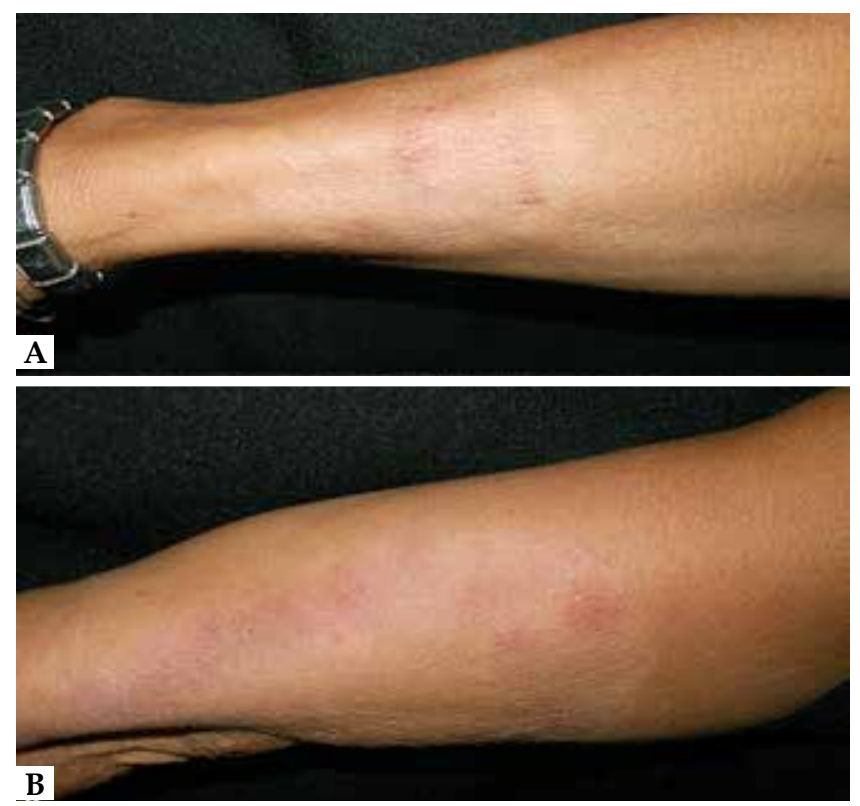

Figure 3: A and B: Clinical aspect of lesions after treatment

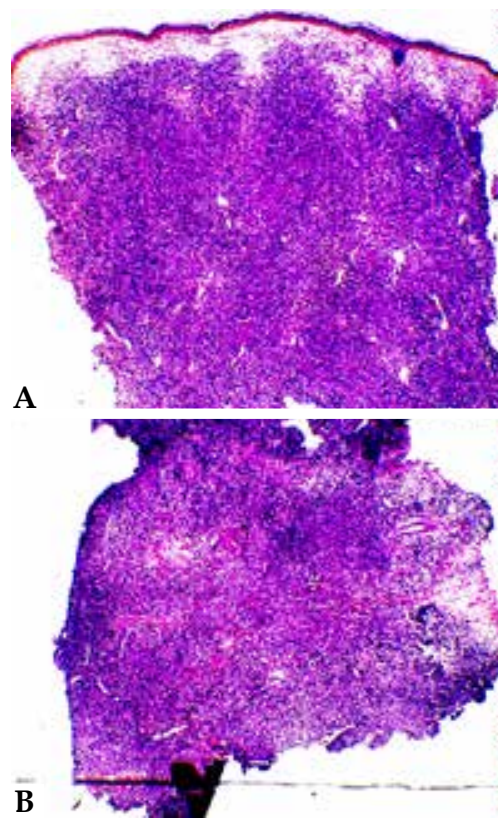

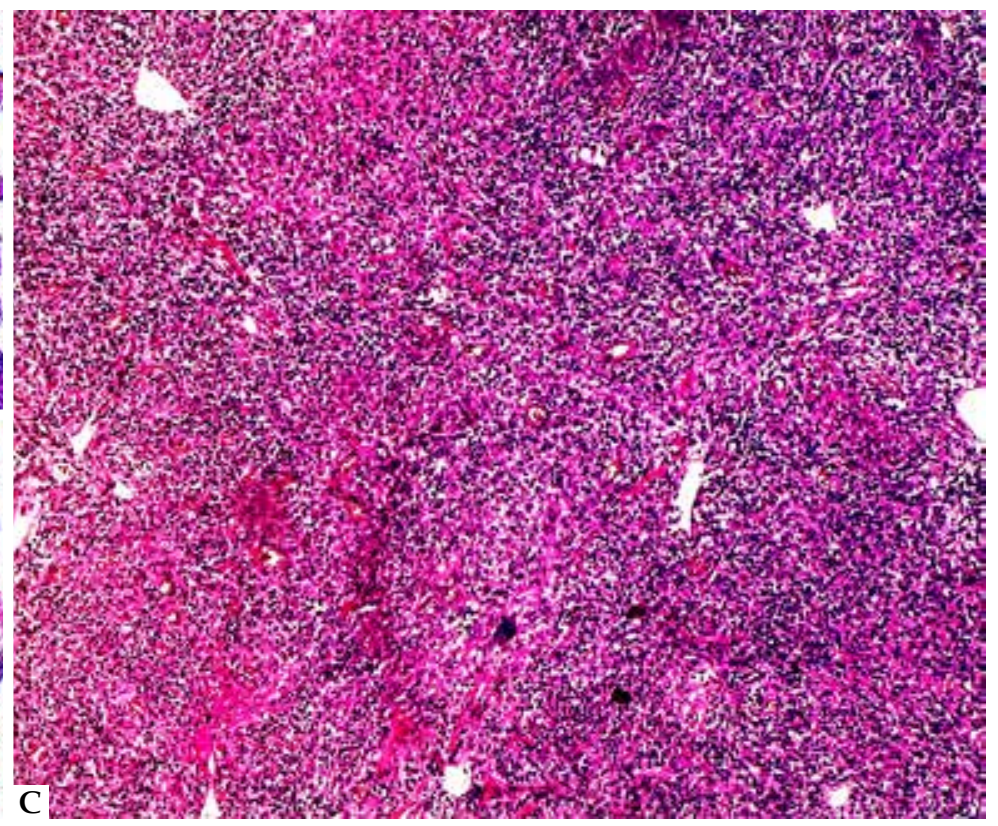

Figure 2: A and B: Anatomopathological examination showed atrophic epidermis and vacuolar interface dermatitis. The dermis and the subcutaneous were filled with a mixed, dense and diffuse inflammatory infiltrate, composed of lymphocytes, histiocytes and abundant plasmocytes, separated from the epidermis by a grenz band. C: Detail of the dense inflammatory infiltrate and vascular proliferation (HE, 400x). 
riasiform plaques or nodules). ${ }^{3}$ It is then classified into two types: gummatous and nodular. The nodular form, as in the present case, consists of painless, hardened erythematous nodules, of varying sizes, which may occur in any site on the skin. They may remain isolated or coalesce to form plaques. Multiple nodules may be distributed in an arciform pattern, with a predilection for the face, interscapular areas and extremities. ${ }^{3}$ The gummatous form presents itself as firm, painless subcutaneous nodules which later develop ulcerations and drainage of necrotic materials.

These two forms, which show great clinical and morphological diversity and show the involvement of the dermis and the subcutaneous by the granulomatous process, may simulate several diseases. ${ }^{4-6}$ Differential diagnosis is broad and includes infectious, inflammatory and neoplastic disorders, such as sarcoidosis, lupus vulgaris, atypical mycobacterial infections, Jorge Lobo's disease, sporotrichosis, leishmaniasis, Hansen's disease, granuloma annulare, pyoderma gangrenosum, lymphoma and pseudolymphoma. ${ }^{3,5,6}$ Spontaneous remission is rare, and consequently, recurrences in the skin become even more destructive with time. In most cases, however, lesions are resolved quickly when treated.

\section{REFERENCES}

1. Revathi TN, Bhat S, Asha GS. Benign nodular tertiary syphilis: a rare presenting manifestation of HIV infection. Dermatol Online J. 2011;17:5.

2. Gjestland T. The Oslo study of untreated syphilis: an epidemiologic investigation of the natural course of the syphilitic infection based upon a study of the BoeckBruusgard material. Acta Derm Venereol Suppl (Stockh). 1955;35:3-368.

3. Pereira TM, Fernandes JC, Vieira AP, Basto AS. Tertiary syphilis. Int J Dermatol. 2007;46:1192-5.

4. Chung G, Kantor GR, Whipple S. Tertiary syphilis of the face. J Am Acad Dermatol. 1991;24:832-5

5. Wu SJ, Nguyen EQ, Nielsen TA, Pellegrini AE. Nodular tertiary syphilis mimicking granuloma annulare. J Am Acad Dermatol. 2000;42:378-80.

6. Erfurt C, Lueftl M, Simon M Jr, Schuler G, Schultz ES. Late syphilis mimicking a pseudolymphoma of the skin. Eur J Dermatol. 2006;16:431-4

7. Varela P, Alves R, Velho G, Santos C, Massa A, Sanches M. Two recent cases of tertiary syphilis. Eur J Dermatol. 1999:9:371-3.

8. Rocha N, Horta M, Sanches M, Lima 0, Massa A. Syphilitic gumma - cutaneous tertiary syphilis. J Eur Acad Dermatol Venereol. 2004;18:517-8.

9. Avelleira JCR, Bottino G. Sífilis: Diagnóstico, tratamento e controle. An Bras Dermatol. 2006:81:111-6.
The histopathologic features of tertiary syphilis lesions consist, in the nodular form, in an abundant plasmocytic infiltrate, with formation of granulomas and many times caseous necrosis. In the syphilitic gumma an important caseous necrosis occurs, surrounded by numerous giant cells and dense inflammatory infiltrate, composed of lymphocytes and plasmocytes, which extends itself to the subcutaneous. ${ }^{3}$ In the case presented, the important density of the infiltrate is highlighted, which, associated with vascular lesions and abundant plasmocytes, led to suspicion of syphilis diagnosis.

Nontreponemal serological tests may present negative results or low titer for late syphilis, specially in cardiovascular or neurosyphilis forms. ${ }^{3,7-9}$ Treatment is carried out with benzathine penicillin at a dose of 2,400,000 IU per week, intramuscular, for three weeks. ${ }^{9}$ In any phase of the disease, the possibility of other sexually transmitted diseases being present must be considered. Thus, serology for HIV and screening for hepatitis B and C ought to be recommended for all patients. ${ }^{3}$ Cases of tertiary syphilis in patients free from HIV have been rarely described in recent years. ${ }^{3-8}$ The case presented emphasizes the need of keeping syphilis in the list of differential diagnoses for long-duration papulonodular lesions, to ensure early diagnostic and treatment, even in the absence of previous history of syphilis or immunosuppression.]

\author{
MAILING ADDRESS: \\ Maraya de Jesus Semblano Bittencourt \\ Avenida Generalíssimo Deodoro, 91 \\ Umarizal \\ 66055-240 - Belém - PA \\ Brazil \\ E-mail:marayabittencourt@hotmail.com
}

How to cite this article: Bittencourt MJS, Brito AC, Nascimento BAM, Carvalho AH, Drago MG. Nodular tertiary syphilis in an immunocompetent patient. An Bras Dermatol. 2016;91(4):528-30. 